\title{
Los mercados orgánicos en México como escenarios de construcción social de alternativas
}

\author{
Héctor Nicolás Roldán Rueda
}

El Colegio de la Frontera Sur, Chetumal, México.

Email: hroldan@ecosur.edu.mx

María Amalia Gracia

El Colegio de la Frontera Sur, Chetumal, México.

Email:magracia@ecosur.mx

\section{María Eugenia Santana}

Universidad Autónoma de Chiapas, San Cristóbal de las Casas, México.

Email: maru.santana@gmail.com

\section{Jorge Enrique Horbath}

El Colegio de la Frontera Sur, Chetumal, México.

Email: jhorbath@ecosur.mx

Resumen: ${ }^{1}$ Ante la evidente manifestación de la crisis civilizatoria por la que atraviesa la humanidad se vienen consolidando propuestas que apuntan a dinamizar y articular sectores excluidos por la economía de mercado. Dentro de estas propuestas, los mercados orgánicos/locales se erigen como iniciativas con la capacidad de incidir en los procesos productivos y en las relaciones sociales que los sustentan, así como también brindar un marco de referencia para recrear otras economías. A partir de un trabajo de investigación realizado en tres mercados orgánicos/locales en México con características heterogéneas se reflexiona sobre los principales elementos que han habilitado su aparición durante la última década y sobre sus potencialidades, límites y tensiones para consolidar otras formas de hacer y pensar los procesos económicos.

Palabras clave: procesos económicos, mercados alternativos, economía solidaria, producción orgánica.

\section{Organic markets in Mexico: scenarios for alternative social construction}

\begin{abstract}
In response to the crisis that the world is facing, some alternative models are emerging aiming to make economic sectors more dynamic and articulate, which usually are excluded by the free market economy. Among these models, organic/local farmer markets are one of the initiatives with the capacity to affect the production process of certain goods and the social networks that sustain them. More over, these types of farmer markets also provide a framework for other
\end{abstract}


forms of economies. Based on fieldwork in three organic/local farmer markets with heterogeneous characteristics in Mexico, this paper considers the principal elements that have enabled the existence of these markets in the last decade. Additionally, this paper analyses these types of farmer market's potentiality, limits, and tensions for consolidating other ways to think and pursue economic processes.

Palabras clave: economic processes, alternative markets, solidarity economy, organic production.

\title{
Mercados orgânicos no México como cenários de construção social de alternativas
}

\begin{abstract}
Resumo:Perante a evidente manifestação da crise civilizacional quevemexperimentando ahumanidade estão sendo consolidadas propostas que visam dinamizar e articularsetores excluídos pela economia de mercado. Dentro dessas propostas, os mercadosorgánicos/locais se erigem como iniciativas com capacidade de influenciar nos processos produtivos e nas relações sociais que os sustentam, bem como proporcionar um quadro de referencia para recriar outras economias. A partir de uma pesquisa realizada em três mercados orgânicos /locais no México, com características heterogêneas, se reflete sobre os principais elementos que permitiram a sua aparição durante a última década e sobre as suas potencialidades, limites e tensões para consolidar outras formas de fazer e pensar processos econômicos.
\end{abstract}

Palavras-chave: processos económicos, mercados alternativos, economia solidária, produção orgânica.

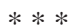

\section{Introducción}

En un contexto de aumento de la producción de productos orgánicos que se caracterizanpor la prescindencia en el uso de insumos de síntesis química ${ }^{2}$, México se ha ido posicionando mundialmente como uno de los principales productores de este sector de la agricultura. Junto al incremento de formas convencionales para comercializarlos -entre las que sobresalen las grandes cadenas de supermercados y las tiendas especializadas- se han generado espacios de intercambio como los mercados locales, que si bien conservan rasgos de los antiguos "tianguis ${ }^{3}$ adquieren distintas significaciones en los nuevos contextos en los que emergen.

En los mercados locales no sólo se comercializan productos orgánicos sino que al promover la eliminación de la intermediación a partir del intercambio directo entre productores y consumidores se exploran distintos tipos de vínculos y relaciones entre los participantes que interrogan sobre las implicaciones y significaciones que tienen para ellos y para los espacios locales donde se crean.

En la última década estas experiencias se han multiplicado; en distintas regiones del país surgieron mercados locales con intereses, preocupa- 
ciones y desafíos comunes. Este artículo busca aportar a la reflexión sobre las condiciones para el fortalecimiento de este tipo de espacios a partir del estudio de tres casos situados en distintos contextos urbanos de México: Playa del Carmen (Quintana Roo), San Cristóbal de las Casas (Chiapas) y Texcoco (estado de México), ciudades con historias y problemáticas distintas ${ }^{4}$.

Para la selección de estos tres casos de estudio se buscó que todos ellos fueran reconocidos como miembros activos de Red Mexicana de Tianguis y Mercados Orgánicos (REDAC); que tuvieran distintos momentos de constitución y promoción de actividades de producción y consumo de productos orgánicos así como diferentes procesos de consolidación y, finalmente, que se insertaran en contextos urbano-regionales con características económicas, sociales y culturales distintas a fin de contrastar su capacidad para responder a los intereses y necesidades locales.

Aun con aspectos comunes, cada una de estas experiencias presenta formas específicas de articulación de motivaciones e intereses así como estrategias para sostenerse en el tiempo logrando mayor visibilidad, autonomía y capacidad de diálogo con otros emprendimientos similares y con actores que inciden directamente en sus actividades (organismos estatales y de la sociedad civil e instituciones académicas).

¿Qué implicaciones y aprendizajes surgen de ellas para pensar otras formas de intercambio? ¿De qué manera se vinculan los distintos actores entre sí y con los territorios en los cuales se instalan los mercados? ¿Qué identidades y formas de participación inauguran?

Exploramos estos interrogantes analizando las potencialidades, tensiones, desafíos y problemáticas que estos procesos conllevan así como las estrategias que adoptan para enfrentarlas a fin de determinar qué tipo de alternativas representan respecto a la producción, al intercambio y al consumo.

Partimos de la premisa de que estas experiencias pueden contribuir a promover alternativas de producción y consumo frente a las propuestas hegemónicas mediante formas directas de intercambios monetarios y no monetarios que van más allá del valor de cambio de las mercancías. Asimismo, asumimos que forman parte de la construcción más amplia de identidades y movimientos sociales que habilitan otros órdenes de poder y de relaciones humanas (Gracia, 2011) en tanto van gestando pautas o modalidades de organización social autogestiva que logran responder a contextos específicos.

\section{Los mercados alternativos en diálogo con teorías, redes y movimientos sociales}

Los mercados orgánicos forman parte de la construcción de alternativas que surgen en diferentes partes del mundo como respuesta a expresiones de la crisis civilizatoria (Toledo, 2012). Entre otras dimensiones de 
dicha crisis, mencionamos el acceso y la seguridad de los alimentos, la concentración de la tierra, los problemas ambientales y la falta de representatividad colectiva (Bartra, 2011; Altieri y Nicholls, 2010; Rosset, 2007).

Desde América Latina se destacan iniciativas ligadas a reivindicaciones sociales, económicas, políticas y ambientales surgidas en comunidades indígenas, afros, campesinas y otros grupos y poblaciones excluidas que han venido luchando por su reconocimiento. Estas experiencias plantean desafíos en función de recrear formas de satisfacer las necesidades humanas más allá de lo material ${ }^{5}$, cuestionando las lógicas de acumulación capitalista y sus efectos en la naturaleza y las relaciones humanas.

Las propuestas colectivas de cambio y transformación social combinan distintos niveles de diálogo y cooperación, así como formas alternativas de percibir la necesidades y prioridades de la sociedad (Coraggio, 2011).

\section{Los diferentes rostros de la producción orgánica: entre la alternativa y la conveniencia}

En el significativo crecimiento mundial de la agricultura orgánica intervienen variados actores así como prácticas económicas, socioproductivas, culturales y políticas diferenciadas. Lo que en un principio se erigió como una alternativa a la industrialización de los sistemas alimentarios y sus efectos en la salud y en el medio ambiente se fue tornando cada vez más un nicho de mercado dentro de un sistema económico en el que sobre todo intervienen grandes corporaciones en detrimento de la producción y el intercambio local.

Por esa razón importa evidenciar hasta qué punto las experiencias que estudiamos tienen la capacidad de generar tejido social y satisfacer así otro tipo de necesidades e intercambios mediante valores que rebasen la mercantilización y permitan transitar del valor de cambio al valor de uso de las mercancías.

En términos competitivos el valor es la cantidad que los compradores están dispuestos a pagar por una mercancía (Porter, 1991). De acuerdo con Simmel (1977) el valor no es una propiedad inherente de los objetos sino que se construye mediante el juicio que los sujetos construyen alrededor de ellos. Guiados por diferentes estímulos, los sujetos van generando procesos internos y colectivos que les permite otorgar "valores" a las mercancías.

El mercado globalizado hace uso de las cadenas productivas por medio de la creación de valor y la minimización de costos. La creación de valor es realizada por diferentes agentes a lo largo de cadenas de valor que imprimen o vuelven a transformar el valor de cambio en un nuevo valor de uso mediante estrategias de comercialización, intermediación y especula- 
ción. Este proceso estimula la producción en función de la demanda y no de las necesidades. Es evidente que hay distintas formas de recuperar el valor de uso de las mercancías: en un caso, acortando los procesos de intermediación (mercados alternativos) y, en el otro, alargándolos y comandando la producción a partir de la demanda y la creación de necesidades (mercado globalizado).

A partir de las entrevistas y revisión de fuentes secundarias identificamos tres grupos ligados a la producción, el consumo y el intercambio de productos orgánicos que involucran distintas formas de articulación con el mercado convencional. En el primero la comercialización se hace por medio de grandes cadenas de supermercados y la producción proviene de empresarios agrícolas con grandes extensiones de tierra, procesos y maquinaria industriales y cuyo objetivo se enfoca hacia el abastecimiento de este tipo de productos al mercado exterior. Este grupo no estimula transformaciones en las bases socioproductivas de la producción agrícola ni fortalece las economías locales dado que responde exclusivamente a las lógicas del mercado y depende de los mercados internacionales donde se inserta, todo lo cual va en detrimento de los principios de la agricultura orgánica (Escalona, 2009; Nelson, et, al, 2011; Gómez, M et, al, 2002).

Un segundo grupo está conformado por pequeños productores organizados mayormente en cooperativas de producción, sociedades de solidaridad social o sociedades de producción rural; si bien en este sector es posible encontrar experiencias que contribuyen con procesos de transformación social al promover la participación e inclusión de sus asociados, la mayor parte de la producción se comercializa a gran distancia del lugar de origen mediante intermediarios. Se refuerza así la dependencia hacia el mercado internacional, y si bien su participación en algunos mercados (como el comercio justo) les permite acceder a sobreprecios que pueden generan mayores beneficios económicos ${ }^{6}$, muchas veces éstos no se ven reflejados en las condiciones de vida, ni de trabajo de los productores locales (Bastos, 2007; Doppler y Gonzales, 2007; García, 2011); este el caso de algunos grupos organizados de pequeños productores de café de Chiapas y Oaxaca.

Finalmente, el tercer grupo comprende pequeños productores locales que participan y promueven, junto con consumidores y otros actores sociales, espacios locales organizados de intercambio. En dichos ámbitos en los que algunas cooperativas venden también una parte de su producción- se plantean maneras de producir, consumir e intercambiar que cuestionan el hecho de que el mercado sea el fin último de las relaciones sociales.

\section{Los procesos económicos en función de la sociedad}

La economía solidaria (ES), como otras propuestas alternativas de producción, consumo e intercambio, promueve relaciones más justas y reivindica las distintas luchas contra la exclusión y la pobreza. La definimos como el conjunto de actividades que contribuyen a la democratización de la economía a partir de compromisos ciudadanos (Laville, 1999). Desde la ES 
se plantean articulaciones sociales que permitan formular estrategias para consolidar proyectos forjados a partir de las particularidades de cada contexto (Fernández, 2001).

Muchos colectivos que se reconocen dentro del campo amplio y en construcción de la ES (Gracia y Horbath, 2014) rigen sus prácticas en función de principios y valores que apuntan a la conformación de otras formas de hacer y pensar la economía, la sociedad y la cultura, lo cual implica otras formas de hacer y pensar la política (Coraggio, 2011). Reconocen que no hay realidad económica necesaria a la que haya que adaptarse o morir y consideran que otras economías son posibles si se parte de una economía empírica (Polanyi, 1974).

Los mercados orgánicos y locales son esfuerzos por explorar nuevas configuraciones socioeconómicas, políticas y culturales en contextos territoriales específicos. Lejos de responder a generalidades (y en ese sentido se oponen diametralmente a la lógica de las franquicias) se adaptan y expresan las motivaciones de sus participantes. No se reducen a expresiones que emergen desde un escenario de pobreza, sino que buscan conectar distintos sectores de la sociedad partiendo de vínculos donde predominan relaciones basadas en principios de reciprocidad, solidaridad e igualdad (Gracia y Horbath, 2014). No todos los participantes acuden guiados por estos principios, ni las relaciones y los procesos escapan a conflictos propios de distintos espacios humanos; para algunos de sus participantes (productores y consumidores) estos espacios no van más allá de una relación comercial, lo cual muestra algunos de los límites y diferentes formas de apropiarse de ellos de acuerdo a intereses particulares (Santana, 2015).

La reproducción de estas experiencias significa considerar que se puede concebir y generar mercados menos concentrados y más justos mediante la inclusión de pequeños productores a las cadenas de intercambio. La principal diferencia entre el sistema de mercado y los mercados locales es que el primero disuelve los lazos de dependencia entre los miembros de una comunidad y fomenta el individualismo (Parry y Bloch, 2000: 4, citado por Santana, 2011) mientras que las experiencias de mercados locales fomentan las relaciones sociales para construir sociedades con mercados y no de mercado o mercantilizadas (Coraggio 2011).

Finalmente, las experiencias que identificamos van más allá de lo económico; la idea de economía social, no significa adjetivar a la economía y agregarle "lo social”" (como si hubiera alguna economía que no estuviera generada a partir de relaciones sociales) sino más bien apunta a que la economía social o social y solidaria ayuda a reconstruir el lazo social dañado por los efectos destructores del mercado y la falta de regulación del Estado (Gracia, 2011). Entendemos el territorio como el espacio que incluye lo político, lo social, lo económico, lo cultural y lo ecológico, un lugar determinado por unas características concretas pero que se ve afectado por los efectos de la globalización (Coraggio, 2011). 


\section{Autonomía y redes de comercio justo}

En los mercados locales se conjugan dos dimensiones de la noción de autonomía que inciden en las prácticas de sus participantes y en los mecanismos que van adoptando estos espacios. La autonomía como proceso individual en el que cada persona tiene la capacidad de tomar sus decisiones en función de satisfacer sus propias necesidades (el consumo, una de ellas) y la autonomía como parte de procesos colectivos donde toma de decisiones se da en función de las necesidades del grupo e implica interdependencia, diálogo y participación.

De acuerdo con Escalona (2009), el campo de acción de los mercados locales es un espacio de política no institucionalizada en el que se fomenta la identidaden términos de diversidad y la autonomía como una forma de reconocer subjetividades históricamente negadas. Así la identidad supone la búsqueda de desarrollo de personasidentificadas con sus raíces y valores culturales que pueden reconocerse y reconocer en los otros una posibilidad de intercambio personal que permite generar distintos tipos de participación individual y colectiva. La autonomía se ubica en un contexto socioespacial que legitima a los grupos excluidos por el sistema, en concordancia con las propuestas de la ES. A partir de ella se reconocen subjetividades, por lo que el lugar y la localidad son el referente geográfico desde el que se ejerce la producción política y espacial de nuevas formas de representación y participación política (Preciado y Uc, 2010).

En estos escenarios, la lucha por la autonomía y las formas de resistencia son flujos, en la medida que "todo movimiento social se configura a partir de aquellos que rompen la inercia social y se mueven, es decir, cambian de lugar, rechazan el lugar al que históricamente estaban asignados dentro de una determinada organización social, y buscan ampliar los espacios de expresión” (Gonçalves, 2001: 81). Su posibilidad de sumarse y articularse con otras experiencias y actores abre espacios físicos y simbólicos de resistencia y supervivencia; buscando sobrevivir comienzan a producir y reproducir sus vidas en forma diferente a como lo propone el capitalismo (Zibechi, 2007).

Un ejemplo es la creación e implementación de las redes de comercio justo bajo un esquema en el cual se pretende articular a diferentes sectores de la sociedad por medio de formas productivas que se diferencian y generan beneficios para los diferente sectores involucrados.

Dentro del comercio justo ${ }^{7}$ existen diferentes enfoques, algunos críticos frente a la economía capitalista y otros que promueven el libre comercio, aunque en general se evidencia que es posible generar mercados, beneficios, intercambios y relaciones exitosos reconociendo el trabajo de las personas.

Las redes de comercio justo realizan un esfuerzo por organizar colectivamente a pequeños productores para generar mayores ingresos, mejorar la representatividad en los intercambios y que exista mayor reconocimiento 
entre los distintos actores. Esto permite participar de nuevos espacios e incluir a nuevos actores, lo que ha demostrado la capacidad reproductiva y pedagógica que contienen sus estrategias (Pérez y Gonzales, 2013).

La noción de justicia en los procesos económicos ha ido adquiriendo un peso importante en los discursos, aunque muchas veces tiene poco efectos en las prácticas, lo que permite cuestionar que aun las acciones más conscientes y bien intencionadas pueden producir resultados opuestos a los buscados (Polanyi, 1974). Por ejemplo, se habla de justicia ambiental pero no se contemplan las emisiones de $\mathrm{CO}_{2}$ producidas por el transporte de alimentos; de justicia social, pero un gran número de productores venden sus productos a intermediarios "justos"; de precio justo, pero en ocasiones pareciera no pasar de su enunciación. Volveremos sobre estos aspectos en el análisis de los resultados.

\section{La producción orgánica en México y la creación de la REDAC ${ }^{8}$}

Diferentes países del mundo han dirigido sus esfuerzos hacia el sector agrícola orgánico, ya sea para satisfacer la demanda interna o como abastecedores del mercado internacional. México se ha ido consolidando como país productor y proveedor de productos orgánicos, y se posiciona como un actor determinante a nivel mundial, según la Federación Internacional de Movimientos de Agricultura Orgánica, IFOAM (2008).

Fuentes de información académicas, e instituciones oficiales, describen el caso de México como la oportunidad de generar ingresos a partir de la exportación de productos orgánicos. El 60\% de la superficie de cultivos orgánicos en México se concentra en Chiapas, Oaxaca y Michoacán, donde predominan pequeñas unidades de producción campesina, además de procesos tradicionales de siembra.

Según el Centro de Investigaciones Interdisciplinarias para el Desarrollo Rural Integral (CIIDRI), en 2008 hubo un aumento en diferentes rubros (superficie, número de productores, empleos directos e ingresos). A pesar del notable aumento en el área cultivada y de los productores involucrados se observa una concentración de los productos cultivados: tan solo 15 (café, aguacate, hierbas y hortalizas) representan el 97.3\% de la superficie con agricultura orgánica, (Gómez y Gómez, 2004).

Estas cifras han colocado en las agendas públicas (tanto del sector gubernamental como iniciativas de distintos actores locales) diferentes estrategias para incentivar, mejorar y estandarizar los procesos de la producción orgánica. El Estado ha manifestado interés en impulsar la producción para la exportación y viene diseñando instrumentos legislativos para la producción, manipulación y empaquetado de los productos orgánicos en detrimento de fortalecer el consumo local (Gómez, et. al, 2002).

Desde los distintos espacios de intercambio local y regional se plantean formas autogestivas y autónomas que buscan alternativas de consu- 
mo y producciónbasadas en valores como la confianza y la reciprocidad. Desde 2004 se ha venido consolidando la Red Mexicana de Tianguis y Mercados Orgánicos (REDAC) como un actor importante en las discusiones y negociaciones de los productores orgánicos del país.

\section{La Red Mexicana de Tianguis y Mercados Orgánicos ${ }^{9}$}

Los tianguis y mercados orgánicos son fruto del esfuerzo de las organizaciones de base enfocadas a la producción orgánica de pequeña escala. Actualmente hay más de 25 mercados orgánicos funcionando y se planea la apertura de otros tantos con el apoyo y la iniciativa de los consumidores vinculados con universidades y organizaciones de la sociedad civil.

La REDAC surge para organizar algunas de las iniciativas que se venían gestando alrededor la producción y el consumo de productos orgánicos en diferentes partes del país. Actualmente está presente en 15 estados como resultado de procesos que cuentan con la participación de diversos actores. Aunque cada mercado se mantienen independiente al tener características propias, todos comparten objetivos comunes: el cuidado por el medio ambiente y aspectos como la justicia económica y social (Nelson, et.al, 2008).

La propuesta es acercar a consumidores y productores para compartir características de los productos, experiencias e historias que se tejen entre los participantes. Estas características, aunadas a la reducción de costos de transporte, empaquetado y la eliminación de los intermediarios persiguen que los pequeños productores logren llegar a más consumidores para ofrecer precios relativamente más asequibles, sin embargo, como veremos más adelante, existen debates que permiten ubicar distintas posiciones respecto al precio final de los productos.

La REDAC apunta a que los mercados locales se construyan con el apoyo de la comunidad para incentivar la producción y el consumo y contribuir al empoderamiento y el fortalecimiento de actores locales, además "apoyar este tipo de vínculos también sirve para la construcción de solidaridad en la comunidad y el establecimiento de relaciones de confianza" (Nelson, et. al, 2008). Las interacciones que se generan entre los participantes vinculados a instituciones académicas, organizaciones civiles y en ocasiones instituciones del gobierno habilitan la inclusión y la diversidad de personas dentro de estos mercados.

Junto a la aceptación y capacidad de convocatoria que han tenido en diferentes regiones, estos espacios enfrentan retos comunes que impiden que reproduzcan a mayor escala y en ocasiones que les cueste alcanzar estabilidad. El principal reto es su capacidad para obtener recursos, la mayoría de los mercados que pertenecen a la red no genera ingresos suficientes que les permitan garantizar las condiciones para funcionar, lo cual ha impedido alcanzar algunos de los objetivos propuestos. 
Otros desafíos y tensiones que enfrenta la red, responde a los marcos regulatorios (leyes, reglamentos y lineamientos) y las implicaciones (mayor control y vigilancia) que tienen sobre las actividades productivas. Las barreras económicas y burocráticas que implica la certificación orgánica hacen que muy pocos productores de pequeña escala la logren obtener. Como respuesta, desde los mercados orgánicos se propusieron procesos de certificación participativa. Este tipo de certificación minimiza la burocracia, no requieren ningún pago de quienes participan de la certificación (productores, consumidores, académicos) y se rige por la combinación de normas oficiales y características de cada región.

\section{La inserción de los mercados en su contexto local: tensiones, diálogos y estrategias}

La viabilidad de estos mercados no puede reducirse a cifras de rentabilidad, ventas y relaciones costo-beneficio. Tampoco se puede pretender que se tome como plausible una propuesta que no es viable en lo inmediato y que, sobre todo, no permite experimentar sus resultados positivos a quienes las encarnan. Por ello, hay que sistematizar y visibilizar las experiencias existentes, de tal manera que más allá de las cifras agregadas puedan dar cuentan mediante otras virtudes de su capacidad y viabilidad (Coraggio, 2011).

Para entender la viabilidad, sin caer el sentido común económico, "es necesario no perder de vista que cuando hablamos de "viabilidad económica” estamos efectuando un juicio de valor mediante el cual comparamos los efectos de las prácticas existentes con ciertos criterios o parámetros estándares deseables [...] Por ello, para poder valorar estas experiencias como otras que manifiestan lógicas económicas que no están sólo regidas por las leyes del mercado, es necesario ir construyendo un concepto de eficiencia alterna que ponga en relación los fines perseguidos y los resultados alcanzados” (Gracia, 2009: 230).

Para efectuar esta tarea en primer lugar presentamos las particularidades de cada mercado y posteriormente analizamos ejes temáticos establecidos previamente al trabajo de campo y otros que emergieron durante su realización.

\section{De las redes de producción y consumo a las redes sociales virtuales. La experiencia de la Miscelánea Orgánica en Playa del Carmen (PdC)}

En medio de la Riviera Maya, cónclave de producción turísticainternacional creado hace algo más de cuarenta años, la Miscelánea Orgánica se ha venido reinventando en función de las posibilidades y dificultades que ejerce la presión del turismo y la falta de apoyos, tanto del gobierno como de otros actores. Sus principales retos pasan por la escasa vocación agrícola que actualmente tiene la región y las dificultades para 
gestionar espacios públicos que les permitan visibilizar, tanto este proyecto como algunas necesidades locales, entre ellas, el acceso a alimentos sanos y de calidad.

Los promotores han desarrollado estrategias que les permite mantenerse activos y vigentes en la REDAC, entre otras, mayor presencia en medios virtuales y la participación en ferias de turismo. Sin embargo, no han logrado poner en acto uno de los principales objetivos de la red: realizar intercambios directos entre los participantes.

“A lo mejor otros tianguis van a pensar que es algo que se está saliendo de la tangente, pero acá es muy particular, tenemos características y no tenemos alternativa, aquí para que consigas que toda la gente descanse un día a la semana al unísono y tenga el tiempo, tres horas en ese día, no se puede, acá la gente trabaja todo el tiempo" (Entrevista a promotora, PdC, marzo 2014).

Como resultado de esta situación no han podido conformar una red de consumidores que permita fomentar los intercambios directos y garantizar la venta de la producción local. Esta característica sólo nos permitió dialogar con los promotores de este mercado ya que durante las visitas no se lograron concretar fechas de encuentros directos entre productores y consumidores. Quienes participan como consumidores son principalmente turistas nacionales o extranjeros que viven por temporadas largas en PdC, quienes realizan pedidos y luego los recogen, sin que existan contactos directos entre ellos y los productores.

"Funcionamos gracias a que hay un mercado cautivo pequeño de clientes 80\%-90\% extranjeros, vienen ya con esa cultura. Entonces si el extranjero se va en verano, son cuatro meses sin ventas, sin nuestros clientes fuertes. Y los que no se fueron de viaje no tiene dinero porque es temporada baja. Además el mexicano oriundo de acá no es muy afín a esto” (Entrevista a promotor, PdC, marzo 2014).

La presencia en medios virtuales les ha permitido estar conectados con otras experiencias y redes. Sin embargo, no han logrado generar vínculos con proyectos afines en la región (Puerto Morelos, Valladolid, Cancún) ${ }^{10}$ como consecuencia de la distancia y los costos, además de las tensiones que la rigurosidad de los aspectos técnicos de la producción orgánica suscita,

"En otro mercado acá en la región, tuvimos la desventaja de que otros productores comenzaron a copiarnos, el problema no es la competencia, el problema es que le hacen creer a la gente que esto es lo mismo pero más barato y de la misma calidad, entonces la gente pierde credibilidad” (Entrevista promotor, PdC, marzo 2014).

Si bien en la cita los promotores hacían referencia exclusivamente a los productos de belleza y cuidado personal que ellos elaboran, también 
mencionaban casos en los que se ofrecían alimentos del mercado convencional dentro de estos espacios. La falta de información permite lacomercialización productos que no han seguido el proceso que supone la producción orgánica, esto trae consecuencias para los pequeños productores, en la medida que sus productos pierden credibilidad y confianza frente al consumidor, además de generar rupturas entre proyectos afines.

Este escenario muestra las tensiones y límites que tienen ciertos contextos para este tipo de experiencias y evidencia los retos en pos de articular una red regional de proyectos similares a fin de crear sinergias y apoyos que circulen y se transformen en herramientas de aprendizaje colectivo.

\section{Repensar lo orgánico. La experiencia del Mercado Artesanal y Agroecológico Comida Sana y Cercana en San Cristóbal de las Casas (SCLC)}

En SCLC se viene desarrollando un proyecto que nació hace nueve años de la iniciativa de cuatro mujeres preocupadas por la calidad y los efectos en la salud de los alimentos regados con aguas negras (situación recurrente principalmente en centros urbanos) y el uso intensivo de pesticidas. De esta inquietud nació la Canasta Orgánica, antecedente de lo que después fue el Tianguis Red de Comida Sana y Cercana y hoy el Mercado Artesanal y Agroecológico Comida Sana y Cercana.

SCLC es una ciudad que reúne habitantes de distintos lugares del país y del mundo. La presencia de comunidades indígenas y su posición geográfica la erigen como un centro de intercambio comercial entre las comunidades aledañas y como una ciudad multicultural que complementa su arquitectura colonial con una diversidad de espacios culturales marcados por la influencia del zapatismo (sin que exista una relación directa con el mercado, es una particularidad del contexto) y las diferentes comunidades indígenas de la región. La presencia de este mercado, no sólo es bien aceptada, sino que además logra generar lazos con otros proyectos afines.

Otras propuestas similares que se articulan dentro de la ciudad y que permiten ampliar el horizonte de esta experiencia son "La Casa del Pan” (allí se pueden encontrar los productos que se ofrecen en este mercado), "Comedor Comunitario La Milpa” (proyecto-restaurante que surgió paralelo al mercado, liderado por una de las fundadoras del tianguis), "Cacao Solidario” (promueven compras comunitarias y sirven de enlace entre productores y consumidores), “Libre Café” (ofrecen café orgánico y otro tipo de productos orgánicos). Al estar enlazados a través de las redes sociales virtuales, logran difundir diferentes propuestas y compartir con su público las actividades de éstas y otras experiencias.

Otra de las instancias de participación colectiva incluye las asambleas en donde se toman decisiones y se ponen en juego las estrategias que se van adoptando. Recientemente, en una asamblea realizada en el 
primer trimestre del 2014, tomaron la decisión de alejarse de la REDAC en función del marco regulatorio que se viene aplicando con la ejecución de la ley de orgánicos (2006) y sus lineamientos (2013).

“Tomamos la decisión de salir de la REDAC porque esta organización está dándonos reglamentos que nosotros no vemos favorables para el tianguis, responden a otros intereses y nosotros queremos ser lo más autónomos posibles con nuestro trabajo y sentimos que los lineamientos de las red no iban a favorecernos" (Entrevista a productor, SCLC, marzo 2014).

Las palabras de uno de los productores son indicativas de que los lineamientos comunes a la red se cuestionan en función de las necesidades e intereses locales. Esta decisión implica ampliar la oferta hacia otros procesos productivos, entre los que se incluyen -además de productos orgánicos que son principalmente hortalizas- productos agroecológicos ${ }^{11}$ (Altieri, 2002), artesanales y locales (cada uno con características particulares, pero con objetivos similares). También ha permitido encontrar espacios y vínculos, que se complementan con el discurso y las prácticas de lo orgánico desde una plataforma más amplia, lo que ha traído efectos tanto positivos como negativos.

Dentro de las virtudes identificamos los alcances de la organización interna en función de los objetivos y los intereses planteados, la vinculación con proyectos afines que no abordan exclusivamente el tema de la producción orgánica y se complementan con propuestas desde la agroecología y la sensación de ser parte de un proyecto más incluyente de otros actores (productores agroecológicos, urbanos, de traspatio). Se han generado tensiones en función de los objetivos y la presenciade alimentos preparados en comparación con los productos agrícolas. Lo que demuestra que no se trata de procesos lineales sino que los participantes van reinventando sus maneras de involucrarse y articularse con el entorno.

De los tres mercados, es el que sesiona más días a la semana. El perfil de los consumidores es diferente en cada día: sábados y miércoles se ubican en un espacio ubicado en el centro y a una cuadra del andador Eclesiástico lo que les permite acercarse a más personas. El sábado es el día en que hay mayor concurrencia, asiste gran número de extranjeros residentes en la ciudad y otros turistas; los miércoles, concurren los “coletos”(en palabras de los participantes, forma en que se conoce a los mestizos nacidos en SCLC) y personas de comunidades indígenas, y los viernes integrantes de la comunidad de El Colegio de la Frontera Sur, donde se instala ese día.

El vínculo que el mercado tiene con el sector académico ha permitido la producción de conocimiento basado en investigaciones ${ }^{12}$. Esto ha contribuido con procesos internos de reflexión y aprendizaje, y ha posibilitado diseñar estrategias de participación para que los tianguistas participen activamente de la toma de decisiones 
“Ahora los tianguistas estamos tomando, no el mando, pero si la toma de decisiones entre todos, antes era entre ellas [las promotoras] los primeros años del tianguis ellas lo hacían, porque fue su proyecto, pero ahora está en un punto en donde tiene que caminar de la mano de los tianguistas y de quienes participan” (Entrevista productor, SCLC, abril de 2014).

La variedad de productos y el número de participantes varía de acuerdo a los días, siendo el sábado cuando más presencia hay de productores (24 en promedio). Dentro de los productos que más se intercambian están las hortalizas, seguidas de los quesos y alimentos preparados que han ido incrementándose (pizza, paella, quesadillas, tostadas, panes, jugos, postres), lo que, frente al número de productores locales, permite pensar en una transformación del objetivo de acercar a productores y consumidores. En voz de un académico que participa como miembro activo de una cooperativa de café en la zona y que estuvo vinculado al mercado, hablando del porque dejaron de participar,

“Al cabo del tiempo la batuta de esto la fueron tomando gente que ya tenía otro sentido que se distanciaba del que tenía en su origen para nosotros, y que empezaban a orientar muy fuertemente el consumo hacia los productos ya elaborados, ya no productores primarios, ya más bien comida elaborada y entonces toda esta idea de que fueran productores, campesinos y acercar al productor se fue cambiando y fueron ganando mucho espacio. Ese espacio ya es todo, menos esa lógica de establecer relaciones cercanas entre quienes producen y quienes consumen”. (Entrevista a miembro de cooperativa de café y académico de Ecosur, SCLC, abril 2014)

Muchos tianguistas son extranjeros, esto ha creado una barrera imaginaria entre los consumidores locales y el lugar. Es muy usual escuchar, a personas locales, que se trata de un lugar para "ricos", o asumir que los precios son muy elevados (los productos orgánicos alcanzan un sobreprecio de 20 a 40 por ciento respecto a su similar convencional, Gómez y Gómez, 2004). El tema de los precios respecto a otros ámbitos de venta de bienes y servicios representa todo un desafío a enfrentar por parte de ésta y otras experiencias pues limita la capacidad de incluir a sectores con distintas capacidades adquisitivas y cuestiona entonces el supuesto de diversidad que pregonan. Por otro lado, la forma en que se asignan los precios, también cuestiona algunos de los supuestos a partir de los cuales nacen las experiencias. Volveremos a este asunto más adelante.

\section{El apoyo institucional como plataforma de consolidación. La experiencia del Tianguis Orgánico de Chapingo}

Este mercado es el más antiguo de los tres, recientemente cumplió once años y ha sido el epicentro -junto con el Círculo de Producción (Jalisco) y el Mercado el Pochote (Oaxaca)-de la creación de la REDAC y de muchas de las actividades promovidas desde la red, por lo tanto muchas de 
las recomendaciones, aprendizajes y conocimientos surgen de aquí. A diferencia de los otros dos mercados nunca ha cambiado su ubicación y abre todos los sábados a un costado de la Universidad Autónoma de Chapingo (UACH), en el Barrio Cooperativo.

Con el apoyo de la UACH se han implementado procesos de conversión de la agricultura convencional a la orgánica con varios productores de diferentes regiones (el estado de México, Oaxaca y Veracruz). La vinculación entre diferentes departamentos de la UACH y el mercado local ha permitido desarrollar actividades (talleres, charlas, visitas a parcelas) entre productores y consumidores para difundir la agricultura orgánica y sus beneficios, lo que a nivel local ha tenido gran recepción y ha vinculado a la población -sobre todo niños, niñas y jóvenes- con algunas de las actividades del tianguis.

Este vinculo ha permitido visibilizar, ante la comunidad universitaria y la población local, el impacto de la agricultura convencional en la salud de la tierra, de los humanos y en el futuro alimentario de las sociedades. A pesar de no estar vinculado directamente con dicha institución, ha ganado un espacio dentro de las actividades académicas y de difusión de problemas y necesidades locales y regionales (como el acceso a alimentos libres de pesticidas y de explotación laboral y ambiental, la inserción de pequeños productores a espacios de intercambio).

Como el mercado de SCLC la presencia de instituciones académicas incide directamente en el desarrollo de las actividades y el éxito de muchas de ellas. En Texcoco también se ubican el Colegio de Posgraduados y el Centro Internacional para el Mejoramiento del Maíz y el Trigo. La presencia de las instituciones académicas incide en la viabilidad de estos mercados y habilita la consolidación de procesos como el de certificación participativa, que en un principio fue liderado por académicos y lentamente ha ido siendo asumido por los tianguistas. La toma de decisiones también ha evolucionando en función de procesos más democráticos, al igual que en SCLC se toman decisiones en asambleas. La cercanía a la academia no ha sido un impedimento en la construcción de formas organizativas propias a cada experiencia.

Existen vínculos entre los estudiantes de la UACH (incluyendo a la preparatoria agrícola) y las actividades del tianguis; muchos de los trabajos de investigación, en diferentes niveles de escolaridad y áreas del conocimiento se vinculan con el tianguis. Ya sea como trabajo de extensión, ofreciendo información a los consumidores, desarrollando tareas que involucran aspectos relevantes de la producción (control de plagas, compostas, rotación de cultivos) o investigaciones sobre los consumidores ${ }^{13}$. Con el acompañamiento de diferentes departamentos de la UACH se ha venido trabajando en la elaboración de insumos orgánicos (foliares, biopreparados, alelopatía, agrohomeopatía) para mejorar los procesos productivos.

La variedad de los productos es similar a la del mercado de SCLC, la principal diferencia se encuentra en que los productores son todos mexica- 
nos. También existe variedad de alimentos preparados, lo que tanto en este mercado como en SCLC constituye una estrategia para atraer nuevos consumidores.

\section{Tejiendo los lazos sociales: motivos, necesidades y vínculos de los consumidores y productores}

El principal motivo para participar como consumidores responde a la preocupación por los efectos sobre la salud de los alimentos regados con aguas negras y agroquímicos y de allí se deriva una segunda motivación: el cuidado del medio ambiente. Sin embargo, a medida que dialogan y se involucran con los productores y sus historias se sienten parte de estos proyectos y apoyan de distintas maneras las actividades que se promueven (certificación participativa, talleres, charlas). Muchos de los consumidores reflexionan y hacen del consumo una práctica más consciente y coherentefrente a las tensiones que surgen del sistema económico, el deterioro ambiental y las relaciones sociales,

"Somos gente que tenemos ganas de cambiar el sistema, de no caer en el consumismo, apoyando empresas grandes, la gente está harta. La forma en que podemos hacer política es saber a quién le compramos, a quién le entregamos el dinero, cómo nos estamos nutriendo, rescatamos muchos saberes tradicionales, muchas cosas que arrastramos. Y vamos a estar haciendo algo siempre, faltan muchísimas cosa por mejorar alrededor de esto, pero vamos en ese proceso" (Consumidor, SCLC, abril de 2014)

"He aprendido muchas cosas, antes era muy inconsciente con lo que consumía, con lo que compraba, como mucha gente, yo a veces decía "bueno qué onda los políticos que hacen mala política” y al final también uno puede hacer política sabiendo a quien le compras, que es lo que estas consumiendo" (Consumidor, Chapingo, mayo 2014).

El hecho de que estos mercados operen como lugar de encuentro entre amigos y familias constituye otro factor de asistencia y genera una respuesta frente a la ausencia sistemática, a partir de la privatización y la violencia, de espacios públicos de interacción y. De ahí que trasciendan los intercambios de productos en tanto tienen capacidad de generar encuentros, diálogos, reflexiones, vínculos, afectos y aprendizajes entre los participantes. Fruto de esto son algunos espacios de discusión en torno a otras formas de consumir, o espacios gastronómicos (cocinas) que permiten intercambiar experiencias o incluso la participación activa en actividades en defensa del maíz criollo, en contra de los transgénicos, reivindicaciones sociales, políticas y alimentarias ${ }^{14}$ como el derecho a tener acceso a alimentos inocuos. 
A pesar de que estos mercados comparten similitudes, existen diferencias que responden al contexto. En Chapingo es más fácil encontrar consumidores que asisten desde hace más de dos años a diferencia de SCLC en donde a pesar de un número importante de consumidores frecuentes es muy usual encontrar nuevos consumidores que están de paso.

Otro aspecto que los diferencia es la información que circula sobre la creación de cada mercado. La estrecha relación de la UACH con el tianguis orgánico como con diferentes esferas de la sociedad ha permitido que se den a conocer desde hace varios años. En una encuesta telefónica realizada por la UACH en 2012 a la población de Texcoco, el tianguis orgánico fue el segundo proyecto que más asociaron a la universidad, después de uno de yerbas medicinales. En SCLC la constante renovación de los participantes no permite que se conozcan información acerca del proceso interno, pero sí se da el interés por conocer detalles acerca de los productos (origen, preparaciones, propiedades), lo cual estimula la confianza entre los participantes y permite que la certificación participativa cobre importancia como forma de garantizar la inocuidad de los productos. Finalmente, en PdC, al no poder consolidar un espacio de intercambio, los consumidores no logran establecer diálogos directos, lo que limita los escenarios para compartir otro tipo de experiencias y de información.

Entre los motivos que les hace interrogarse por el origen de los productos la primera razón es poder asegurarse de que sean orgánicos. Para ello, no necesariamente se exige un sello de certificación, hay otros elementos en los que reparan los consumidores, por ejemplo, la presentación, el tamaño y el sabor de los productos así como conocer la procedencia y el proceso por la propia voz de los productores. También se comparten aspectos como las formas de controlar plagas, alimentos de temporadas, usos medicinales y preparaciones

Respecto a los precios existen diferentes opiniones dentro de los consumidores. Entre los inconvenientes que identifican los consumidores para continuar realizando las compras en estos mercados se identifican la variedad y el precio en conjunto, como los principales factores de decisión.

Las categorías que refieren al "precio justo" se agotan en la disposición a pagar teniendo en cuenta las características de los productos y los vínculos que se van creando con los productores. En ninguno de los tres mercados estudiados los precios se colocan en función de un análisis de los costos de la producción sino que suasignación se calcula en función de los precios del mercado convencional. ¿Cuál es entonces la noción de precios justos que rodea estos escenarios? ¿Son justos porque son más caros? A partir de la investigación no encontramos que se valore, por ejemplo, el precio más elevado en función del cuidado del medio ambiente, el costo de los insumos orgánicos o el esfuerzo de trabajo que representa para los productores y sus familias. En este sentido es claro que aún se necesita discutir y definir la noción de justicia dentro de estas experiencias, reconocer qué implica o supone la justicia en términos de intercambio, tanto en lo 
que respecta a los productores como a los consumidores pues el término sólo parece ser un lema que en la práctica opera como un justificante para subir los precios respecto al mercado convencional.

Retomando a Simmel (1958), Santana (2009, 2015), observa que la noción de precio justo depende de las ventajas subjetivas que los participantes le otorgan a un producto; como consecuencia de esto el intercambio sucede cuando las dos partes llegan a un acuerdo mediante procesos de negociación cara a cara (regateo) que los beneficien, especialmente, para que personas de menos recursos económicos puedan acceder a estos productos sin que dejen de haber diferenciaciones en los precios para que quienes puedan pagar lo hagan. En la medida que no exista espacio para negociar, estaremos ante un mercado de precios fijos en donde el consumidor está sujeto a su restricción presupuestaria para poder participar del intercambio, recreando así un modelo de intercambio que no se aleja -y pareciera que reprodujera- uno de los fallos del mercado capitalista al poseer gran poder de mercado, emulando las estrategias monopolísticas.

Existen algunos esfuerzos incipientes por generar intercambios no monetarios, sin embargo a pesar de que los consumidores declaran estar interesados en poder intercambiar libros, juguetes o ropa por productos, los intercambios son aislados y no muy frecuentes.

Los consumidores vinculan estos mercados con las características de sus productos (sobre todo destacan las palabras fresco-limpio-saludable) y con las posibilidades que les abren en términos de sociabilidad (se repiten las palabras amigos-convivencia-agradable). Esto muestra que además de productos orgánicos asociados con los términos referidos se abre una experiencia que mediante la ampliación de espacios de encuentro puede ayudar a modificar hábitos y costumbres.

En cuanto a los motivos de los productores para hacer la conversión de la agricultura convencional a la orgánica, se destaca la influencia de algún familiar o vecino, el beneficio económico de comercializar los productos en espacios diferenciados y el cuidado del medio ambiente, además del apoyo y asesoría de los productores y académicos, principalmente, durante el proceso de transformación de los cultivos. La falta de apoyos financieros y las distancias para poder llegar hasta las parcelas para recibir apoyo técnico influyen de manera negativa.

Los productores que en el caso de las hortalizas, frutas y hierbas son generalmente campesinos de áreas rurales de cada región, así como quienes ofrecen alimentos preparados, declaran que con el tiempo la participación en estos mercados va encontrando otros sentidos, como la convivencia, la amistad y la conciencia respecto a las generaciones futuras. Estos nuevos sentidos se van adquiriendo a raíz de los diálogos entre productores y consumidores, sus preocupaciones, las formas de producir, el interés por las familias o el simple hecho de un saludo cálido, va configurando estos escenario en espacios de convivencia, en donde el producto es un 
elemento más de las virtudes del lugar.

“Lo más importante es la conexión que tenemos entre todos, tenemos más amistad y conocemos a nuestros clientes, nos platican, te dicen buenas días o ¿cómo estás?, el convivio entre nosotros, entre compañeros y consumidores. Me siento mejor que en mi casa, porque en la casa estoy aburrida y acá platicamos y si tiene uno problemas igual te aconsejan, ha sido muy buena experiencia”. (Entrevista productora, SCLC, abril 2014).

"Es una muy buena experiencia, estar ofreciendo los productos que uno mismo cultiva e interactuar con los clientes y más que clientes ya son amigos y cada vez que ves que vienen pues nos saludamos ya de beso y abrazo, esa confianza que tenemos es lo interesante de este lugar” (Entrevista productor, Chapingo, mayo 2014).

A pesar de las dificultades y contradicciones, estas expresiones de los participantes permiten pensar que el encuentro contribuye a transformar lógicas y comportamientos individuales y colectivos. La importancia de las interacciones entre los participantes habilita espacios de discusión en donde se reconocen límites, virtudes y alcances para tratar de conseguir los fines perseguidos y continuar formulando estrategias que les permitan continuar vigentes.

A manera de resumen presentamos el siguiente cuadro comparativo:

\section{Cuadro 1. Cuadro comparativa mercados seleccionados.}

\begin{tabular}{|c|c|c|c|}
\hline & Playa del Carmen & San Cristóbal de Las Casas & Chapingo \\
\hline Tiempo & 6 años & 9 años & 11 años \\
\hline $\begin{array}{l}\text { Ubicación y } \\
\text { horario }\end{array}$ & $\begin{array}{l}\text { No cuentan con un espacio ni } \\
\text { horario definido. }\end{array}$ & $\begin{array}{l}\text { Miércoles y Sábado de } 10 \text { am a } 3 \text { pm: } \\
\text { Avenida } 16 \text { de Septiembre } 32 \text {, Barrio } \\
\text { Mexicanos } \\
\text { Viernes de } 10 \mathrm{am}-3 \text { pm ECOSUR }\end{array}$ & $\begin{array}{l}\text { Sábado de } 10 \mathrm{am} \text { a } 3 \mathrm{pm}: \text { Calle } \\
\text { Igualdad S/N Colonia Pueblo } \\
\text { Cooperativo }\end{array}$ \\
\hline Productores & $\begin{array}{l}\text { No existe un numero de } \\
\text { productores constante. }\end{array}$ & 27 & 28 \\
\hline $\begin{array}{l}\text { Caracteristicas } \\
\text { consumidores }\end{array}$ & $\begin{array}{ll}\text { Extranjeros residentes y } \\
\text { turistas. Muy pocos } \\
\text { consumidores locales. }\end{array}$ & $\begin{array}{l}\text { Extranjeros residentes en la ciudad, } \\
\text { turistas, residentes y en ocasiones } \\
\text { personas de comunidades } \\
\text { cercanas. Consumidores asociados a } \\
\text { la academia }\end{array}$ & $\begin{array}{l}\text { Personas residentes en Texcoco } \\
\text { y áreas de influencia cercana al } \\
\text { Distrito Federal. Consumidores } \\
\text { asociados a la academia }\end{array}$ \\
\hline Promotores. & $\begin{array}{l}\text { Productores de productos de } \\
\text { belleza y cuidado personal. }\end{array}$ & $\begin{array}{l}\text { A raiz un proceso de reestructuración } \\
\text { en la organización interna, son los } \\
\text { mismos tianguistas quienes han } \\
\text { tomado el control del lugar. }\end{array}$ & $\begin{array}{l}\text { Un reciente proceso de } \\
\text { organización ha permitido que } \\
\text { los tianguistas asuman las } \\
\text { actividades. Investigadores y } \\
\text { estudiantes de la UACH estan } \\
\text { muy comprometidos y siguen } \\
\text { de cerca los diferentes procesos. }\end{array}$ \\
\hline $\begin{array}{l}\text { Fijación } \\
\text { precios }\end{array}$ & $\begin{array}{l}\text { - Se toma como referencia el } \\
\text { precio en el mercado } \\
\text { convencional } \\
\text { - No existe un mecanismo para } \\
\text { calcular los precios }\end{array}$ & $\begin{array}{l}\text { - Se toma como referencia el precio } \\
\text { en el mercado convencional } \\
\text { - No existe un mecanismo para } \\
\text { calcular los precios }\end{array}$ & $\begin{array}{l}\text { - Se toma como referencia el } \\
\text { precio en el mercado } \\
\text { convencional } \\
\text { - No existe un mecanismo para } \\
\text { calcular los precios }\end{array}$ \\
\hline $\begin{array}{l}\text { Categorias de } \\
\text { discurso } \\
\text { recurrentes }\end{array}$ & $\begin{array}{l}\text { - Comercio justo - justicia } \\
\text { - Solidaridad } \\
\text { - Reciprocidad } \\
\text { - Respeto }\end{array}$ & $\begin{array}{l}\text { - Comercio justo } \\
\text { - Solidaridad } \\
\text { - Reciprocidad } \\
\text { - Respeto }\end{array}$ & $\begin{array}{l}\text { - Comercio justo - justicia } \\
\text { - Solidaridad } \\
\text { - Reciprocidad } \\
\text { - Respeto }\end{array}$ \\
\hline
\end{tabular}

Fuente: Elaboración propia con datos de trabajo de campo. 


\section{Conclusiones}

Del trabajo realizado emergen cuatro aspectos que permiten sintetizarlo y ofrecer reflexiones finales. El primero tiene que ver con la producción y el consumo como prácticas que contribuyen a gestar procesos colectivos de toma de decisiones. El segundo, la posibilidad de explorar otras formas de intercambiar; el tercero con la articulación conotras propuestas que permiten el diálogo y la retroalimentación y, por último, la formación de identidades con valores y principios que cuestionan prácticas y lógicas hegemónicas.

En primer lugar y basados en los aportes de autores como Coraggio (2011); Razeto (1999); Gudynas (2011) y Gracia (2011), identificamos que en ellas hay una búsqueda y predominancia de formas colectivas para gestionar los procesos económicos. Los casos analizados surgieron de la iniciativa de consumidores con preocupaciones y necesidades similares que, mediante diferentes formas de construir vínculos, van transformando la visión y las experiencias de cada actor con el intercambio, al tiempo que se van reconociendo tensiones y en ocasiones distanciamientos de otras experiencias.

Estos procesos aportan a la construcción de esferas de poder social con la capacidad de dar respuesta a problemas que los aquejaban (acceso a productos sanos, intercambios directos, cuidado del medio ambiente). Los protagonistas operan como agentes de transformación a nivel local al poner en práctica formas de producir, consumir e intercambiar que suponen explorar distintos procesos de organización así como la capacidad de generar articulaciones con emprendimientos y grupos afines.

El incentivo hacia otras formas de producir y consumir va ayudando a que emerjan alternativas para intercambiar mediante diferentes mecanismos de inclusión y participación colectiva. Ello contribuye con la construcción de espacios que, sin negar la existencia del mercado y de sus instrumentos de cooptación, reproducen otras formas de intercambios.

Observamos la capacidad que tienen los espacios para detonar luchas en función de la relación que se crea con la comunidad. Ejemplo de ellos son los vínculos que se establecen con instituciones académica, la presencia en espacios de discusión y participación ciudadana y actividades en la defensa del medio ambiente. Al tiempo que identificamos tensiones que habilitan procesos de aprendizaje colectivo y el diseño de estrategias que permiten adaptarse a los constantes cambios de cada contexto.

Finalmente, otra de las virtudes que se pueden resaltar es su aporte a la conformación de identidades mediante prácticas y valores que ponen en cuestión lógicas y dinámicas hegemónicas, abriendo la posibilidad a transformaciones graduales que favorezcan la reproducción de escenarios de solidaridad, justicia y respeto, mediante el reconocimiento del otro como parte esencial del proceso. 


\section{Notas}

${ }^{1}$ El estudio se basa en los resultados de investigación para realizar la tesis titulada " $L a$ construcción social de los mercados alternativos en México. Estudio de tres casos en distintos contextos y procesos de consolidación” para obtener el grado de Maestro en Ciencias de El Colegio de la Frontera Sur, generación 2013-2014. Agradecemos a la Dra. Birgit Schmook, Dra. Minerva Arce Ibarra y al Dr. Fernando Limón, investigadores de El Colegio de la Frontera Sur por los comentarios y sugerencias realizados.

${ }^{2}$ La producción orgánica ha desencadenado múltiples definiciones, todas coinciden en que ésta supone la eliminación de insumos químicos y el riego con aguas negras durante el proceso de producción, además de incluir aspectos de carácter ambiental y social.

${ }^{3}$ El tianguis es el mercado local o regional donde se reúnen los productores directos, agricultores o artesanos, a intercambiar sus productos. Si bien, hoy en día los tianguis conservan en ciertos aspectos exteriores su aire tradicional (vendedores sentados en la calle, regateo, etc.) son uno de los nervios centrales de la articulación de la economía mercantil simple o campesina. (Paré, 1975: 85)

${ }^{4}$ El trabajo de campo se realizó entre los meses de febrero y mayo de 2014 a partir de una guía de observación, encuestas a consumidores (40), entrevistas semiestructuradas a productores, promotores y académicos vinculados con estas experiencias (15) y diálogos informales con los participantes. Se participo en encuentros entre promotores que pertenecen a la red que articula a los mercados y visitas a productores orgánicos en el estado de Oaxaca en una estancia académica en la Universidad Autónoma de Chapingo durante el mes de mayo.

${ }^{5}$ Los conceptos tradicionales de desarrollo apuntaron a la necesidad de subsistencia pensando que el ser humano solamente debe sobrevivir en términos materiales, al respecto otros autores han aportado al a construcción analítica de otro tipo de necesidades y sus satisfactores (Max Neef, et al., 1993; Boltvinik, 2007)

${ }^{6}$ En muchas ocasiones se trata de la apropiación de un discurso por parte de los intermediarios para generar mayores excedentes, no siempre los sobreprecios del mercado justo o de algunas de las otras formas de obtener sobreprecios contribuyen con las comunidades productoras, generalmente la producción es pagada por un intermediario a un precio mayor que el precio del mercado convencional. Lo que se traduce en la no redistribución equitativa de las ganancias.

${ }^{7}$ El comercio justo se compone diferente elemento, entre los que destacamos las relaciones directas de los productores, evitando intermediarios, el pago por un precio justo, las relaciones de confianza y respeto entre los participantes y el cuidado del medio ambiente y la salud. (Consultar Caracciolo (2014)

${ }^{8}$ Un panorama ampliado de la producción orgánica en México se podrá encontrar en "Espacios de intercambio local de productos orgánicos en México. Panorama, tensiones y procesos de aprendizaje”Roldan y Gracia, en prensa.

${ }^{9}$ El sitio oficial de la red es

${ }^{10}$ Pese a las características del contexto pudimos observar cómo a lo largo de la región 
se están reproduciendo, por fuera de la red, otras experiencias similares.

${ }^{11}$ Los principios básicos de la agroecología incluyen: reciclaje de nutrientes y energía, sustitución de insumos externos; mejoramiento de la materia orgánica y la actividad biológica del suelo; diversificación de las especies de plantas y recursos genéticos de los agroecosistemas en tiempo y espacio; integración de los cultivos con la ganadería, la optimización de las interacciones y la productividad del sistema agrícola en su totalidad, en lugar de los rendimientos aislados de las distintas especies (Gliessman 1998).

${ }^{12}$ Entre los temas abordados se encuentran propuestas relacionados con la producción orgánica y los espacios de intercambio, las representaciones sociales de los productos orgánicos, o redes en torno a determinados productos. En el sitio en internet de la red de bibliotecas de Ecosur se pueden encontrar tesis y artículos

${ }^{13}$ Ver http://www.chapingo.mx/scru/agrohomeopatia/xlvi.html

${ }^{14}$ En muchas ocasiones sirven para reintroducir en la dieta de los consumidores productos que tradicionales olvidados (moringa, chaya, variedades de chiles, piñón mexicano, entre otros) por diferentes razones (consumismo, homogenización de la dieta basada en cereales, proteína animal y algunas hortalizas tradicionales). 


\section{Bibliografía}

Altieri, M. (2002), Agroecology: the science of natural resource management for poor farmers in marginal environments. Agriculture, Ecosystems and Environment, Berkeley.

Altieri, M,. y Nicholls, C (2010), “Agroecología: Potenciando la agricultura campesina para revertir el hambre y la inseguridad alimentaria en el mundo”, en Revista de Economía Crítica, No 10, Madrid.

Bartra, A. (2011), “Hambre, dimensión alimentaria de la gran crisis”, en Revista Mundo Siglo XXI, № 26, México.

Bastos, M. (2007), "La justicia del comercio. Un análisis critico de los fundamentos del comercio justo”, en Revista de investigaciones políticas y sociológicas, Vol 6, Santiago de Compostela.

Boltvinik, J. (2007), “Autodeterminación y florecimiento humano. Reflexiones sobre desarrollo, política social y pobreza”, en Empleo, ingreso y bienestar, Vol.11, México.

Caracciolo, M. (2014), “Economía Social y Solidaria: mercados y valor agregado en actividades rurales y urbanas”, en Economía solidaria y cuestión regional en Argentina de principios de siglo XXI, entre procesos de subordinación y prácticas alternativas, Buenos Aires.

Coraggio, J. (2009), “Karl Polanyi y la otra economía en América Latina”, en ¿Qué es lo económico? Materiales para un debate necesario con el fatalismo. Buenos Aires.

Coraggio, J. (2011), Economía social y solidaria. El trabajo antes que el capital, Abya-Yala, Quito.

Doppler, F. y Gonzales, A. (2007), “El comercio justo. Entre la institucionalización y la confianza”, en Problemas de desarrollo. №149, México.

Escalona, M. (2009), Los tianguis y mercados locales de alimentos ecológicos en México: su papel en el consumo, la producción y la conservación de la biodiversidad y cultura, Tesis de doctorado en Agroecología y Desarrollo Rural Sustentable, Instituto de Sociología y Estudios Campesinos, Córdoba, España.

Federación Internacional de Movimientos de Agricultura Ecológica, IFOAM (2008), Los principios de la agricultura orgánica, En línea, consultado el 10-11-2014:

http://www.ifoam.org/sites/default/files/ poa_folder_spanish.pdf

Fernández, R. (2001), “Capitalismo global, resistencias sociales y estrate- 
gias de poder”, en Radicalizar la democracia, Verbo divino, Pamplona.

García, A. (2011), “El comercio justo: ¿una alternativa de desarrollo local?”, en Polis $N^{\circ}$, Polis Revista Latinoamericana, Santiago de Chile.

Gliessman, S. (1998), Agroecology: ecological process in sustainable agriculture. Ann Arbor Press, Ann Arbor, MI.

Gómez, M., Gómez, L., y Schwentensius, R. (2002), “Dinámica del mercado internacional de productos orgánicos y las perspectivas para México”, en Revista Momento Económico, México.

Gómez, L., y Gómez, M. (2004), La agricultura orgánica en México: Un ejemplo de incorporación y resistencia a la globalización. Manuscrito no publicado, Oaxaca.

Gonçalves, P. (2001), Geo-grafías: movimientos sociales, nuevas territorialidades y sustentabilidad, México.

Gracia, M. (2009), “Organizaciones productivas autogestionadas: propuestas para la economía desde el trabajo”, en Cuadernos del GACE, Santa Marta.

Ibíd, (2011), Fábrica de resistencias y recuperación social: Experiencias de autogestión del trabajo y la producción Argentina. El Colegio de México AC, México.

Gracia, M. y Horbath, J. (2014), “Un recorrido por las experiencias de trabajo asociativo autogestionado en el sur de México”, en Cuadernos de desarrollo rural, 11(73), Bogotá.

Gudynas, E. (2011), “Caminos para las transiciones post extractivistas”, en Transiciones. Post extractivismo y alternativas al extractivismo en Perú, RedGE - CEPES, Lima.

Laville, J. (1999), “Economía solidaria, economía social, tercer sector: las apuestas europeas”, en Biblioteca Virtual TOP sobre Gestión Pública. Disponible en línea, consultado el 100-11-2014: http://www. top. org. ar/ecgp/ FullText/000000/LAVILLE

Max-Neef, M., Elizalde, A., y Hopenhayn, M. (1994), Desarrollo a escala humana: conceptos, aplicaciones y algunas reflexiones, Icaria, Santiago de Chile.

Nelson, E.; Schwentesius, R.; Gómez, L. y Gómez, M. (2008), “Un movimiento orgánico local que crece: La Red Mexicana de Tianguis y Mercados Orgánicos”, en LEISA. Revista de agroecología, Lima.

Paré, L. (1975), “Tianguis y economía capitalista”, en Nueva Antropolo- 
gía. Revista de Ciencias Sociales, México.

Pérez, A., y González, A. (2013), “Contradicciones del comercio justo en México”, en Del sabor a café y sus nuevas invenciones. Escenarios cafetaleros de México y América Latina, México.

Polanyi, K. (1974), La gran transformación. Critica del liberalismo económico, Madrid.

Porter, M. (1991), La ventaja competitiva de las naciones, Vergara, Buenos Aires.

Preciado y Uc, (2010), La construcción de una geopolítica crítica desde América Latina y el Caribe. Hacia una agenda de investigación regional, en Geopolítica(s) 2010, vol. 1, 65-94, México.

Razeto, L. (1999), “La economía de solidaridad: Concepto, realidad y proyecto”, en Persona y Sociedad, Santiago de Chile.

Rosset, P. (2007), “Mirando hacia el futuro: La reforma agraria y la soberanía alimentaria”, AREAS Revista Internacional de Ciencias Sociales, № . 26, Murcia.

Santana, M. (2011), “Los mercados alternativos y la economía solidaria”, en Revista Electrónica de Ciencias Sociales, № 16, Paraíba.

Ídem (2015), Tianguis alternativos: alcances y dificultades, En prensa.

Simmel (1977), Filosofía del dinero, Madrid.

Toledo, V. (2012), “Diez tesis sobre la crisis de la modernidad”, en Polis, $N^{\circ}$ 33, PolisRevista Latinoamericana de Ciencias Sociales, Santiago de Chile.

Zibechi, R. (2007), Autonomías y emancipaciones: América Latina en movimiento. Universidad Nacional Mayor de San Marcos, Fondo Editorial de la Facultad de Ciencias Sociales.

Recibido: 03.12.2014

Aceptado: 08.06.2015 